\title{
How We Treat Genitourinary Cancers During COVID-19 Pandemic?
}

\section{Deepak Dabkara ${ }^{1}$, Sandip Ganguly ${ }^{1}$, Joydeep Ghosh ${ }^{1}$, Amol Patel ${ }^{2}$, Atul Batra ${ }^{3}$, Chandan Krushna Das ${ }^{4}$, TVSVGK Tilak ${ }^{5}$, Bivas Biswas ${ }^{1}$}

${ }^{1}$ Department of Medical Oncology, Tata Medical Center, Kolkata, India. ${ }^{2}$ Army Hospital Research \& Referral, New Delhi. ${ }^{3}$ Department of Medical Oncology, All India Institute of Medical Sciences, New Delhi, India. ${ }^{4}$ Regional Cancer Center, Post Graduate Institute of Medical Education and Research, Chandigarh, India. ${ }^{5}$ Armed Forces Medical College. Pune, India.

\begin{abstract}
Coronavirus disease (COVID-19) caused by severe acute respiratory syndrome - coronavirus 2 (SARS-CoV-2) has become a pandemic and affected the entire globe. Daily routine life is affected due to global restriction of movement and infected more than 3.7 million peoples in more than 200 countries. It's an invisible enemy which has imposed a clear threat to the humanity. Patients other than COVID-19 is also suffering a lot due to this unforeseen circumstances. This pandemic also shaken the powerful nations with best of healthcare settings. Oncology is a unique sector of healthcare as cancer patients are at more risk of SARS-CoV-2 infection and cancer treatments are also affected. Many regulatory bodies and professional organizations have come up with guidelines for healthcare personals and patients to guide anti-cancer treatment during COVID-19 pandemic. It's time to formulate local treatment guidelines to guide cancer treatment with optimum use of healthcare resources at society and national level keeping in mind the load of COVID-19 at the concerned region. We have formulated guidelines to manage genitourinary cancer patients during this pandemic, especially in resource constraint setting with the aim of - optimum treatment of these patients with reduction of risk of contracting SARS-CoV-2 infection without affecting oncological outcome.
\end{abstract}

Keywords: SARS-CoV-2- gentourinary- COVID-19- guidelines- cancer

Asian Pac J Cancer Care, 5 (Suppl 1), 147-152

\section{Introduction}

Coronavirus disease (COVID-19) caused by severe acute respiratory syndrome - coronavirus 2 (SARS-CoV-2) has become a pandemic and posed a great threat to humanity. With over 200 countries affected by the pandemic, more than 3.7 million infected cases, and deaths exceeding 250,000 as on $8^{\text {th }}$ May 2020 [1], the impact of COVID-19 pandemic is enormous in terms of loss of human lives and financial disruption, which is leading to loss of employment.

Cancer patients are at an increased risk of acquiring the infection, requiring ventilator support and a higher risk of death. Patients who received chemotherapy or underwent surgery in a month before acquiring SARS-CoV-2 infection were at high risk of an adverse outcome [2-3]. Thus, the scenario in oncology is unique
Submission Date: 05/26/2020 Acceptance Date: 06/28/2020

\footnotetext{
Corresponding Author:

Dr. Bivas Biswas

Department of Medical Oncology, Tata Medical Center, Kolkata, India

Email: bivasbiswas@gmail.com
}

and patients with cancer are facing a clear danger during the COVID-19 pandemic. There are increased chances of disease recurrence due to delay in anti-cancer treatment.

The oncologists face the following situations in clinical practice -a) Patient with cancer and symptomatic SARS-CoV-2 infection; b) Cancer patients tested positive for SARS-CoV-2 in contact screening, but asymptomatic and c) Asymptomatic cancer patient with undetermined SARS-CoV-2 status. Patients in oncology outpatient clinics can be from the following categories - a) those who are under evaluation for suspected cancer or recently diagnosed with cancer, b) those who are already on active anticancer treatment, c) those who are on follow-up after completion of active anticancer treatment. Further, the underlying cancer can be either localized or locally 
advanced where intention of treatment is cure or metastatic disease where intention of treatment is palliative. The questions that needs to be addressed while treating patients with genitourinary cancers (GUC) during this pandemic are -

(1) Localized disease -Whom to treat now and for whom treatment can be deferred without compromising the oncological outcomes? Is anti-cancer treatment safe during this pandemic? And can alternative strategy act as a bridge to the deferred curative therapy?

(2) Metastatic disease: Who should be treated without delay and whose treatment can be deferred? How much treatment is safe and optimal? Any alternative safe approach e.g. less toxic but effective?

(3) How to consult patients who are on follow up and to minimize their hospital visits.

We formulated a guideline for management of GUC patients during the current COVID-19 pandemic with the primary aim of providing optimum cancer treatment during the current country-wide lockdown without compromising oncological outcomes and minimizing risk of SARS -CoV-2 infection to our patients and health care staff.

\section{Urothelial tract cancers}

\section{Superficial bladder cancer}

Most of the patients ( $75 \%$ ) with bladder cancer present as superficial bladder tumors. High grade non-muscle invasive cancer can be treated with trans-urethral resection of bladder tumor [TURBT] +/- intravesical BCG and cystoscopy surveillance. Patients with low-grade superficial bladder tumors can be treated with a single dose of immediate intravesical gemcitabine after TURBT [4]. The risk of getting SARS-CoV-2 infection is much higher than the benefit of intravesical BCG if a patient has to attend a health care facility frequently.

\section{Muscle invasive bladder cancer/upper urothelial tract cancers}

Cisplatin based neoadjuvant chemotherapy (NACT) followed by radical cystectomy is the standard treatment for muscle invasive bladder cancer in cisplatin eligible patients. Gemcitabine- cisplatin [GC] should be considered as the regimen of choice due to lesser toxicity and similar efficacy [5], despite absence of a phase 3 randomized control trial [RCT] over MVAC (Methotrexate, vinblastine, doxorubicin, cisplatin) and dose dense MVAC (ddMVAC).

Prior studies (before the NACT era) have shown that delaying bladder cancer surgeries by a few weeks is detrimental and may lead to worse outcomes [6]. But during this pandemic, it may be worthwhile to defer surgery for 4-6 weeks for relatively asymptomatic patients and those with incidental diagnosis. A phase III RCT suggested that delaying chemotherapy till relapse did not result in worse survival as compared to immediate chemotherapy post-cystectomy [7]. For patients who underwent upfront surgery and have pT3 /pT4, N0 or N1 disease, adjuvant chemotherapy can be deferred for at least
90 days without compromising the outcome.

\section{Advanced/metastatic disease}

A newly diagnosed patient with suspected metastatic disease will require blood tests to check organ functions and computed tomography (CT) of chest and whole abdomen as baseline staging evaluation. A biopsy or fine needle aspiration is necessary for confirmation of diagnosis before starting treatment. Patients with Eastern Cooperative Oncology Group (ECOG) performance status (PS) 0 to 1 and who are cisplatin eligible we use 3-weekly gemcitabine ( $1000 \mathrm{mg} / \mathrm{m}^{2}$ Day 1 and Day 8 ) and cisplatin $\left(70 \mathrm{mg} / \mathrm{m}^{2}\right.$ Day 1 only) regimen with growth factor support, to the patients who can maintain adherence to treatment owing to restriction of movements during this pandemic. This will reduce the hospital visits as compared to recommended gemcitabine-cisplatin 4 weekly cycles wherein gemcitabine is given on D1, D8, D15 and cisplatin is administered on D2. Dose dense MVAC should be avoided in view of higher toxicity and comparable efficacy with the gemcitabine - cisplatin regimen. If possible, day 8 chemotherapy can be given at a community health center to avoid frequent visits. Cisplatin ineligible patients will be offered gemcitabine - carboplatin [8]. Patients with Eastern Cooperative Oncology Group performance status (ECOG PS 2, 3 and 4) should be offered palliative care. Gemcitabine induced lung injury, though rare, can mimic symptoms of COVID -19 and should be kept in mind [9].

Immune check-point inhibitors [ICIs] are approved as first-line treatment in platinum ineligible patients and can be used in patients with high PD-L1 score. It should be clearly discussed that only a few percentage of patients get a prolonged response with anti PD-L1 therapy. Patients who are already on anti PD L1 therapy and have achieved a good response can consider a treatment break and restart therapy upon disease progression or after the pandemic is controlled. Pembrolizumab can be given $400 \mathrm{mg}$ every 6 weeks as per the latest USFDA approval and nivolumab can be given $480 \mathrm{mg}$ every 4 weekly as opposed to routine cycles and thus hospital visits can be reduced by $50 \%$.

Five ICIs are approved in $2^{\text {nd }}$ line treatment of urothelial tract cancer, out of which nivolumab, atezolizumab, durvalumab and pembrolizumab are available in India. However, the benefit is limited to few patients only. ICIs can cause pneumonitis, which might be difficult to differentiate from SARS-CoV-2 infection and treatment of both is drastically different. Patients should be warned of this side effect and upon developing any such symptoms the patient should contact the nearest health care facility immediately. ICIs remain the drug of choice after platinum failure and are relatively well tolerated with few grade 3 or 4 ICIs induced pneumonitis. The third and subsequent line of treatment should be avoided as the standard of care is not available in India and risk-benefit ratio is high for any experimental therapy during this pandemic. If a patient is responding clinically, imaging may be deferred for 3 to 4 cycles.

\section{Prostate Cancer}

Prostate cancer is a disease of older adults, who 
often have other comorbid conditions. They are at high risk of acquiring SARS-CoV-2 infection and optimum care should be given to them during this pandemic. There are certain subsets of prostate cancer patients where treatment can be delayed or the regimen can be altered without compromising cancer related outcomes.

\section{Localized prostate cancer}

Prostate cancer is generally a slowly progressing disease, with low and intermediate risk disease amenable for radical prostatectomy, radical radiotherapy as well as active surveillance, on a case to case basis. The question is - how long can we delay the surgery? Korets et al [10] in their study on 1561 men with localised prostate cancer opting for surgery, concluded that a delay of $>60$ days was not associated with any adverse pathological outcomes. Additionally, it did not correlate with worse biochemical recurrence free survival. Therefore, patients can be re-assured that delaying treatment in the current scenario, would not adversely affect their outcomes.

Another clinical dilemma is regarding the surgical approach- whether it should be an open or a minimally invasive surgery? The potential benefits of minimally invasive (robotic/laparoscopic) surgery include lesser blood loss, well visualised operative field, lesser postoperative discomfort and lesser in-hospital stay. However, there have been realistic concerns regarding the risk of dissemination of SARS-CoV-2 during minimally invasive surgery, which is considered an aerosol generating procedure [11]. Particles in surgical smoke have been demonstrated to contain a variety of toxic and virulent materials potentially capable of infecting through inhalation. In a nutshell, a surgical delay for patients with localised prostate cancer may not be very harmful, and if needed- surgery should preferably be performed via an open approach. Adjuvant radiotherapy if indicated can be delayed till recurrence. If radical radiotherapy is planned, then hypofractionation [once weekly x 5-6 weeks] should preferably be used.

\section{Locally advanced prostate cancer}

Patients planned for definitive radiotherapy plus androgen deprivation therapy should be started on neoadjuvant ADT. Neoadjuvant ADT may be safely given for 4-6 months. Consider use of 3 or 6 monthly formulations over monthly injection. Hypofractionated external beam radiotherapy should be used and may be delayed up to 6 months.

\section{Metastatic hormone sensitive prostate cancer [mHSPC]}

The treatment of $\mathrm{mHSPC}$ has witnessed a paradigm shift in the last few years. Androgen deprivation therapy (ADT) alone is the treatment of choice for a minority of mHSPC. ADT with chemotherapy (docetaxel) or androgen receptor (AR) targeted therapy (abiraterone acetate, enzalutamide, apalutamide) is the new standard of care in majority of mHSPC patients [12-13]. Aim of therapy in the present time is to minimize hospital visits without compromising oncological outcomes. For symptomatic patients, use of GnRH antagonist is preferred, which is rapid acting and also minimizes the risk of testosterone flare. Once initiated, patients should be encouraged to take further injections at peripheral centres and follow up 3-4 monthly. For asymptomatic patients, GnRH analogues may be considered, which can be used at 3 or 6 monthly intervals. Surgical castration is better avoided. Interim follow-up can be done by telemedicine.

It is prudent to avoid chemotherapy during this pandemic as there are higher chances of myelosuppression, febrile neutropenia and resultant morbidity. Amongst all available trial results of ADT with other agents in patients with $\mathrm{mHSPC}$, enzalutamide remains the safest and should be first choice during this pandemic followed by abiraterone acetate-prednisolone. Chemotherapy should be considered as the least preferable option and if required, can be delayed up to 4 months [14]. Follow-up intervals should be increased to 2-3 months and patients can be monitored telephonically with local lab tests. Radiological tests should be postponed unless there is some urgent clinical indication like, cord compression or fracture.

\section{Castrate resistant prostate cancer}

Various factors including patient's age, comorbidities, ECOG PS, duration of response to prior treatment and disease burden determine the choice of therapy. The available options include chemotherapy (docetaxel, cabazitaxel), AR targeted therapy (abiraterone acetate, Enzalutamide), poly ADP ribose polymerase (PARP) inhibitors (olaparib) for those with germline BRCA/ ATM mutations, and ICIs for those with tumor positive for microsatellite instability. Chemotherapy, olaparib and immunotherapy may be avoided due to associated myelosuppression and immunosuppression. AR targeted therapy should be preferred because they are less toxic, require less frequent monitoring and fewer hospital visits. Patients who have progressed on multiple lines of therapy and are symptomatic should be offered hospice care at a local health facility or oral cyclophosphamide [15]. Bone modifying agents, like - zoledronic acid can be given at 3 monthly intervals.

\section{Renal Cell Cancer (RCC)}

\section{Early stage disease}

Radical nephrectomy remains the treatment of choice from stage 1 to stage 3 renal cell cancers and should be practiced. Patient can be kept on close observation in small size tumor $(<2 \mathrm{~cm})$ or surgery can be delayed for few weeks in relatively asymptomatic patients. Open surgery can be preferred over laparoscopic surgery to avoid aerosol generation. Alternative approach like radiofrequency ablation or transarterial embolization can be attempted in place of radical surgery in small tumors or those with borderline fitness. Adjuvant use of tyrosine kinase inhibitors [TKI] should be discouraged in view of minimal survival advantage with very high treatment related toxicities.

\section{Metastatic disease- upfront therapy}

Last decade has seen a significant change in the 
management of metastatic clear cell renal cell cancer (RCC). Vascular endothelial growth factor (VEGF) targeted therapies and very recent ICIs +/- vascular endothelial growth factor (VEGF) targeted therapies have become the new standard first-line therapy for mRCC [16-18]. In addition to systemic imaging, all patients should be assigned a risk category (favourable, intermediate and poor) based on International Metastatic RCC Database Consortium criteria which includes Karnofsky PS, haemoglobin, platelet count, absolute neutrophil count, corrected calcium, and time from diagnosis to systemic therapy.

A recent phase III randomized controlled trial demonstrated that sunitinib alone was not inferior to sunitinib followed by cytoreductive nephrectomy in mRCC. However, there is a role of cytoreductive nephrectomy in a small subset of patients (in those with oligo-metastatic disease, very low burden disease or with indolent course). During the ongoing pandemic, it is prudent to delay cytoreductive nephrectomy.

Either VEGF targeted therapy or ICIs should be considered as the first line therapy in clear cell mRCC. Recent studies have shown pembrolizumab + axitinib, avelumab + axitinib, ipilimumab + nivolumab and bevacizumab + atezolizumab to have superior efficacy to sunitinib. Complete response rates are higher with these agents compared to VEGF targeted agents alone. Specific susceptibility to bacterial or viral infections in patients receiving ICI have not been studied. There is a possibility that patients undergoing ICI based therapy could be more immunocompetent than cancer patients undergoing chemotherapy. Also there is a possibility of cytokine release syndrome with use of ICI which can account for higher COVID-19 related complications [19]. Pembrolizumab can be used $400 \mathrm{mg}$ every 6 weeks and nivolumab can be used $480 \mathrm{mg}$ every 4 weeks as per the latest USFDA recommendation [20]. There can be overlapping features of cytokine storms due to ICI and SARS-Cov-2 infection. Thus, the decision to use ICI over VEGF TKI should be carefully discussed with patients. Use of therapy will require more hospital visits, but has a higher chance of complete response with long-term control. At minimum, for elderly, frail and patients with multiple comorbidities - it may be judicious to use VEGF targeted therapy, while for others, immunotherapy combination / VEGF targeted therapy are the options.

\section{2nd line \& subsequent line of therapy}

Patients who have progressed on the first line anti VEGF TKI may be treated with nivolumab, lenvatinib + everolimus or axitinib. Another active agent, cabozantinib is not available in India. Among these, axitinib or nivolumab [4 weekly schedule] might be better options because lenvatinib +evverolimus is associated with higher toxicity, and dose reduction is needed in approximately $45 \%$ of patients. Further, everolimus is immunosuppressive and can cause interstitial lung disease, mimicking the symptoms of COVID-19.
Testicular germ cell tumors

Testicular germ cell tumors (TGCT) are the most common solid organ cancers in males between the age of 15-40 years. These are highly curable malignancies in all stages. Since the intention of treatment is cure in all stages, it is imperative to adhere to the standard treatment.

Some of the changes / precautions which can be considered in treatment for GCT patients during this period

1. For stage 1 seminoma, instead of active surveillance, a single cycle of carboplatin with Area Under Curve x 7 can be preferred. This will require less frequent hospital visits and will not compromise the efficacy.

2. In advanced stages, avoid etoposide, ifosfamide and cisplatin [VIP] based chemotherapy as it is more myelosuppressive than EP or BEP.

3. Bleomycin toxicity can mimic symptoms of Covid -19. For standard risk and intermediate risk disease, etoposide \& cisplatin [EP] should be the treatment of choice. For high risk disease, there is a trade-off between bleomycin, etoposide \& cisplatin [BEP] and VIP

In conclusions, the following principles of treatment should be used during treatment of patients with GUC during this COVID -19 pandemic.

1. Delay any treatment whenever feasible if oncological outcome is not compromised.

2. To avoid exposure, minimize hospital visits. Use telecommunication in the form of telephonic consultation or telemedicine.

3. Avoid chemotherapy and immunosuppressive therapy, whenever feasible. Try to find an alternative.

4. Shortest duration external beam radiotherapy regimen may preferably be used for prostatic RT.

5. For patients receiving oral targeted therapies, lab tests can be done at local labs and can be consulted on telephone or telemedicine.

6. Targeted therapy is safer and should be preferred over chemotherapy during COVID-19 pandemic.

7. Surgery may be delayed whenever possible.

8. Open approach instead of minimally invasive surgery should be preferred.

9. Germ cell tumors should not be denied chemotherapy due to high cure rates.

10. Clinic based routine follow-up should be discouraged.

11. Prophylactic growth factors should be used liberally with chemotherapy.

12. Radiological response assessment preferably delayed if there is a clinical response.

13. Screening \& enrolment in clinical trials should be carefully executed during this pandemic if the patient (s) can maintain adherence to the protocol and in close $\&$ constant collaboration of the study team, sponsor and the ethics committee.

14. All patients \& health care staff should follow the universal precautions laid down by local \& national health authorities to prevent contracting SARS-CoV-2 infection. 


\section{Acknowledgements}

Funding

None

\section{Conflicts of Interests}

None to declare for each author

\section{Declaration of authorship}

All authors fulfils the criteria of authorship as per contribution and journal policy requirement.

\section{References}

1. "WHO Coronavirus Disease (COVID-19) Dashboard." n.d. Accessed May 9, 2020 [Available from: https://covid19. who.int/.

2. Liang W, Guan W, Chen R, Wang W, Li J, Xu K, Li C, Ai Q, Lu W, Liang H, Li S, He J. Cancer patients in SARSCoV-2 infection: a nationwide analysis in China. The Lancet Oncology. 2020 03;21(3):335-337. https://doi.org/10.1016/ s1470-2045(20)30096-6

3. Onder G, Rezza G, Brusaferro S. Case-Fatality Rate and Characteristics of Patients Dying in Relation to COVID-19 in Italy. JAMA. 202003 23;. https://doi.org/10.1001/ jama.2020.4683

4. Messing EM, Tangen CM, Lerner SP, Sahasrabudhe DM, Koppie TM, Wood DP, Mack PC, Svatek RS, Evans CP, Hafez KS, Culkin DJ, Brand TC, Karsh LI, Holzbeierlein JM, Wilson SS, Wu G, Plets M, Vogelzang NJ, Thompson IM. Effect of Intravesical Instillation of Gemcitabine vs Saline Immediately Following Resection of Suspected Low-Grade Non-Muscle-Invasive Bladder Cancer on Tumor Recurrence. JAMA. 201805 08;319(18):1880. https://doi. org/10.1001/jama.2018.4657

5. Jian D, Yu C, Hequn C, Jinbo C, Feng Z, Xiongbing Z. Gemcitabine/cisplatin versus methotrexate/vinblastine/ doxorubicin/cisplatin for muscle-invasive bladder cancer: A systematic review and meta-analysis. Journal of Cancer Research and Therapeutics. 2018;0(0):0. https://doi. org/10.4103/0973-1482.188434

6. Lee CT, Madii R, Daignault S, Dunn RL, Zhang Y, Montie JE, Wood DP. Cystectomy Delay More Than 3 Months From Initial Bladder Cancer Diagnosis Results in Decreased Disease Specific and Overall Survival. Journal of Urology. 2006 04;175(4):1262-1267. https://doi.org/10.1016/s00225347(05)00644-0

7. Sternberg CN, Skoneczna I, Kerst JM, Albers P, Fossa SD, Agerbaek M, Dumez H, de Santis M, Théodore C, Leahy MG, Chester JD, Verbaeys A, Daugaard G, Wood L, Witjes JA, de Wit R, Geoffrois L, Sengelov L, Thalmann G, Charpentier D, Rolland F, Mignot L, Sundar S, Symonds P, Graham J, Joly F, Marreaud S, Collette L, Sylvester R. Immediate versus deferred chemotherapy after radical cystectomy in patients with pT3-pT4 or N+ M0 urothelial carcinoma of the bladder (EORTC 30994): an intergroup, open-label, randomised phase 3 trial. The Lancet Oncology. 2015 01;16(1):76-86. https://doi.org/10.1016/s14702045(14)71160-x

8. De Santis M, Bellmunt J, Mead G, Kerst JM, Leahy M, Maroto P, Gil T, Marreaud S, Daugaard G, Skoneczna I, Collette S, Lorent J, de Wit R, Sylvester R. Randomized Phase II/III Trial Assessing Gemcitabine/Carboplatin and Methotrexate/Carboplatin/Vinblastine in Patients With Advanced Urothelial Cancer Who Are Unfit for Cisplatin-
Based Chemotherapy: EORTC Study 30986. Journal of Clinical Oncology. 201201 10;30(2):191-199. https://doi. org/10.1200/jco.2011.37.3571

9. Chi D, Brogan F, Turenne I, Zelonis S, Schwartz L, Saif M. Gemcitabine-induced pulmonary toxicity. Anticancer research. 2012;32(9):4147-9.

10. Korets R, Seager CM, Pitman MS, Hruby GW, Benson MC, McKiernan JM. Effect of delaying surgery on radical prostatectomy outcomes: a contemporary analysis. BJU International. 2011 Nov 16;110(2):211-216. https://doi. org/10.1111/j.1464-410x.2011.10666.x

11. Cohen SL, Liu G, Abrao M, Smart N, Heniford T. Perspectives on Surgery in the Time of COVID-19: Safety First. Journal of Minimally Invasive Gynecology. 2020 05;27(4):792-793. https://doi.org/10.1016/j.jmig.2020.04.003

12. Fizazi K, Tran N, Fein L, Matsubara N, Rodriguez-Antolin A, Alekseev BY, Özgüroğlu M, Ye D, Feyerabend S, Protheroe A, De Porre P, Kheoh T, Park YC, Todd MB, Chi KN. Abiraterone plus Prednisone in Metastatic, Castration-Sensitive Prostate Cancer. New England Journal of Medicine. 201707 27;377(4):352-360. https://doi. org/10.1056/nejmoa1704174

13. Davis ID, Martin AJ, Stockler MR, Begbie S, Chi KN, Chowdhury S, Coskinas X, Frydenberg M, Hague WE, Horvath LG, Joshua AM, Lawrence NJ, Marx G, McCaffrey J, McDermott R, McJannett M, North SA, Parnis F, Parulekar W, Pook DW, Reaume MN, Sandhu SK, Tan A, Tan TH, Thomson A, Tu E, Vera-Badillo F, Williams SG, Yip S, Zhang AY, Zielinski RR, Sweeney CJ. Enzalutamide with Standard First-Line Therapy in Metastatic Prostate Cancer. New England Journal of Medicine. 201907 11;381(2):121131. https://doi.org/10.1056/nejmoa 1903835

14. Sweeney CJ, Chen Y, Carducci M, Liu G, Jarrard DF, Eisenberger M, Wong Y, Hahn N, Kohli M, Cooney MM, Dreicer R, Vogelzang NJ, Picus J, Shevrin D, Hussain M, Garcia JA, DiPaola RS. Chemohormonal Therapy in Metastatic Hormone-Sensitive Prostate Cancer. New England Journal of Medicine. 201508 20;373(8):737-746. https://doi.org/10.1056/nejmoa1503747

15. Ganguly S, Dabkara D, Biswas B, Ghosh J. Metronomic therapy in metastatic castrate-resistant prostate cancer: Experience from a tertiary cancer care center. Indian Journal of Cancer. 2018;55(1):94. https://doi.org/10.4103/ ijc.ijc_346_17

16. Motzer RJ, Penkov K, Haanen J, Rini B, Albiges L, Campbell MT, Venugopal B, Kollmannsberger C, Negrier S, Uemura M, Lee JL, Vasiliev A, Miller WH, Gurney H, Schmidinger M, Larkin J, Atkins MB, Bedke J, Alekseev B, Wang J, Mariani M, Robbins PB, Chudnovsky A, Fowst C, Hariharan S, Huang B, di Pietro A, Choueiri TK. Avelumab plus Axitinib versus Sunitinib for Advanced Renal-Cell Carcinoma. New England Journal of Medicine. 201903 21;380(12):1103-1115. https://doi.org/10.1056/ nejmoa 1816047

17. Motzer RJ, Tannir NM, McDermott DF, Arén Frontera O, Melichar B, Choueiri TK, Plimack ER, Barthélémy P, Porta C, George S, Powles T, Donskov F, Neiman V, Kollmannsberger CK, Salman P, Gurney H, Hawkins R, Ravaud A, Grimm M, Bracarda S, Barrios CH, Tomita Y, Castellano D, Rini BI, Chen AC, Mekan S, McHenry MB, Wind-Rotolo M, Doan J, Sharma P, Hammers HJ, Escudier B. Nivolumab plus Ipilimumab versus Sunitinib in Advanced Renal-Cell Carcinoma. New England Journal of Medicine. 201804 05;378(14):1277-1290. https://doi.org/10.1056/ nejmoa1712126

18. Rini BI, Plimack ER, Stus V, Gafanov R, Hawkins R, 
Nosov D, Pouliot F, Alekseev B, Soulières D, Melichar B, Vynnychenko I, Kryzhanivska A, Bondarenko I, Azevedo SJ, Borchiellini D, Szczylik C, Markus M, McDermott RS, Bedke J, Tartas S, Chang Y, Tamada S, Shou Q, Perini RF, Chen M, Atkins MB, Powles T. Pembrolizumab plus Axitinib versus Sunitinib for Advanced Renal-Cell Carcinoma. New England Journal of Medicine. 201903 21;380(12):11161127. https://doi.org/10.1056/nejmoa1816714

19. Xu Z, Shi L, Wang Y, Zhang J, Huang L, Zhang C, Liu S, Zhao P, Liu H, Zhu L, Tai Y, Bai C, Gao T, Song J, Xia P, Dong J, Zhao J, Wang F. Pathological findings of COVID-19 associated with acute respiratory distress syndrome. The Lancet Respiratory Medicine. 2020 04;8(4):420-422. https:// doi.org/10.1016/s2213-2600(20)30076-x

20. Research, Center for Drug Evaluation and. 2020. "FDA Approves New Dosing Regimen for Pembrolizumab." FDA, April. https://www.fda.gov/drugs/drug-approvalsand-databases/fda-approves-new-dosing-regimenpembrolizumab.

\section{c) (7) (8)}

This work is licensed under a Creative Commons AttributionNon Commercial 4.0 International License. 